M. TANABE

KODAI MATH. J.

18 (1995), 284-294

\title{
AUTOMORPHISMS OF RAMIFIED COVERINGS OF RIEMANN SURFACES
}

\author{
Masaharu TANABE
}

\section{Introduction}

Let $M, N$ be Riemann surfaces, $\pi: M \rightarrow N$ be an unlimited covering map, and $T \in \operatorname{Aut}(M)$. The purpose of this paper is to study the existence of some $T_{*} \in \operatorname{Aut}(N)$ which makes the following diagram commutative.

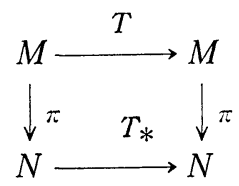

In general, it is not always the case that there exists a $T_{*} \in \operatorname{Aut}(N)$ which satisfies $\pi \circ T=T_{*} \circ \pi$. We will exhibit in section 3 an example such that there is no $T_{*} \in \operatorname{Aut}(N)$ which satisfies $\pi \circ T=T_{*} \circ \pi$. There are few papers concerning this problem, and the author can find only a paper by Martens [5] which gives a necessary and sufficient condition for the existence of $T_{*} \in \operatorname{Aut}(N)$ when $M, N$ are compact Riemann surfaces in terms of homology groups. In this paper, we do not assume that Riemann surfaces $M, N$ are compact. We assume that a covering map $\pi: M \rightarrow N$ is unlimited, ramified, finite-sheeted, and $T \in$ $\operatorname{Aut}(M)$ fixes all of the ramification points of $\pi$. We say that $(M, \pi, N)$ is of excluded type if $N=C$ and the image set of ramification points of $\pi$ consists of one point on $N$. We shall show

THEOREM. Let $\pi: M \rightarrow N$ be an unlimited, ramified, finite-sheeted covering, and of non-excluded type. Then there exist a Riemann surface $S$ and unlimited covering maps $\sigma: M \rightarrow S$ and $q: S \rightarrow N$ such that $\pi=q \circ \sigma$, where $q$ is unramified, and for any $T \in \operatorname{Aut}(M)$ fixing all of the ramification points of $\pi$ there exists a $T_{p} \in \operatorname{Aut}(S)$ making the following diagram commutative.

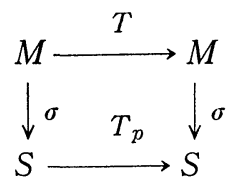

Received May 10, 1994; revised August 2, 1994. 
As corollaries of this theorem, we have

COROLLARY 1. Let $\pi: M \rightarrow N$ be an unlimited, ramified, l-sheeted covering, and of non-excluded type. Suppose that $l$ is a prime number. Then for an arbitrary $T \in \operatorname{Aut}(M)$ fixing all of the ramification points of $\pi$, there exists a $T_{*} \in$ $\operatorname{Aut}(N)$ which satisfies $\pi \circ T=T_{*} \circ \pi$.

COROLLARY 2. Let $\pi: M \rightarrow N$ be an unlimited, ramified, finite-sheeted covering, and of non-excluded type. Suppose that $N$ is simply connected. Then for an arbitrary $T \in \operatorname{Aut}(M)$ fixing all of the ramification points of $\pi$, there exists $a T_{*} \in \operatorname{Aut}(N)$ which satisfies $\pi \circ T=T_{*} \circ \pi$.

In the assertion of the theorem, $q: S \rightarrow N$ is unramified, thus the problem whether $T$ can be projected to $\operatorname{Aut}(N)$ or not is reduced to the case that the covering map is unramified.

The auther wishes to express his gratitude to Professor Shiga for his patient guidance and Dr. Matsuzaki for suggesting this problem and valuable suggestions.

\section{Preliminaries}

Let $M, N$ be Riemann surfaces and $\pi: M \rightarrow N$ be a non-constant holomorphic map. We call the triple $(M, \pi, N)$ a covering surface and also call $M$ a covering surface of $N$. For each $x \in M$, there exist a local coordinate $z$ vanishing at $x$, a local coordinate $\zeta$ vanishing at $\pi(x)$, and an integer $n>0$ such that $\pi$ is given by $\zeta=z^{n}$. If $n>1, x$ is called a ramification point of order $n$. We call $n$ the ramification number of $x$. Let $b_{\pi}(x)=n-1$ and call it the branch number of $x$. We call $(M, \pi, N)$ an unlimited covering provided that for every curve $\gamma$ on $N$ and every point $x$ with $\pi(x)=\gamma(0)$, there exists a lift $\gamma^{*}$ on $M$ with the initial point $x$. We say that $(M, \pi, N)$ is of excluded type if $N=C$ and the image set of ramification points of $\pi$ consists of one point on $N$.

Definition 1. Let $(M, \pi, N)$ be a covering surface. By $\operatorname{Aut}_{\pi}(M)$, we denote the subset of $\operatorname{Aut}(M)$ each element of which satisfying that, for every ramification point $x \in M, \pi(x)=\pi \circ T(x)$ and $b_{\pi}(T(x))=b_{\pi}(x)$.

It is easy to see that if $T \in \operatorname{Aut}(M)$ fixes all of the ramification points, then $T \in \operatorname{Aut} \pi(M)$.

From now on, we assume a covering $(M, \pi, N)$ is unlimited and finitesheeted. For a point $x_{i} \in N$, let $\left\{x_{i k}^{*}\right\}=\pi^{-1}\left(x_{\imath}\right)$ and $r\left(x_{i k}^{*}\right)$ be the ramification number of $x_{i k}^{*}$. For each image of ramification points $x_{i} \in N$, we denote by $\nu_{x_{2}}$ a common multiple of $r\left(x_{i k}^{*}\right)$ (not necessary the least common multiple). To each $x_{i k}^{*} \in \pi^{-1}\left(x_{\imath}\right)$, we assign a number $\nu_{x \imath} / r\left(x_{i k}^{*}\right)$. We define the characteristic $\chi$ given by

$$
\chi=2 \gamma-2+\Sigma_{i}\left(1-1 / \nu_{x_{i}}\right)+n_{\infty}
$$

whenever $N$ is of finite type with genus $\gamma$ and $n_{\infty}$ punctures. Otherwise $\chi=\infty$. 
The following theorem is called the limit circle theorem of Klein and Poincaré (see [1]). It plays a significant role in the sequel.

TheOREM (Klein and Poincaré). Let $M$ be a Riemann surface and $\left\{x_{1}, x_{2}\right.$, ... a discrete sequence on $M$. To each point $x_{k}$ we assign the symbol $\nu_{k}$ which is an integer $\geqq 2$ or $\infty$. If $M=\widehat{\boldsymbol{C}}(=\boldsymbol{C} \cup\{\infty\})$ we exclude two cases;

1) $\left\{x_{1}, x_{2}, \cdots\right\}$ consists of one point and $\nu_{1} \neq \infty$.

2) $\left\{x_{1}, x_{2}, \cdots\right\}$ consists of two points and $\nu_{1} \neq \nu_{2}$.

Let $M^{\prime}=M-\cup_{\nu_{k}=\infty}\left\{x_{k}\right\}, M^{\prime \prime}=M-\cup_{k}\left\{x_{k}\right\}$. Then there exists a simply connected Riemann surface $\tilde{M}$, a discontinuous group $G$ selfmappings of $\tilde{M}$ such that

a. $\tilde{M} / G \simeq M^{\prime}, \tilde{M}_{G} / G \simeq M^{\prime \prime}$, where $\tilde{M}_{G}$ is $\tilde{M}$ with the fixed points of the elliptrc elements of $G$ deleted, and

b. the natural projection $\pi: \tilde{M} \rightarrow M^{\prime}$ is unramified except over the points $x_{k}$ with $\nu_{k}<\infty$ where $b_{\pi}(\hat{x})=\nu_{k}-1$ for all $\hat{x} \in \pi^{-1}\left(\left\{x_{k}\right\}\right)$.

Further, $G$ is uniquely determined up to conjugation in the full group of automorphisms of $\tilde{M}$. The conformal type of $\tilde{M}$ is uniquely determined by the characteristic of the data: that is, by the genus $g$ of $M$ and the sequence of integers $\left\{\nu_{1}, \nu_{2}, \cdots\right\}$. (If we set $\chi=2 g-2+\sum_{j}\left(1-1 / \nu_{j}\right)$, then

$$
\begin{aligned}
& \chi<0 \Longleftrightarrow \tilde{M} \simeq \hat{\boldsymbol{C}} \\
& \chi=0 \Longleftrightarrow \tilde{M} \simeq C \\
& \chi>0 \Longleftrightarrow \tilde{M} \simeq \Delta \quad \text { (unit disk), here } \chi \text { may be } \infty \text {.) }
\end{aligned}
$$

Using this theorem we have

LEMMA. Let $(M, \pi, N)$ be an unlimited, finite-sheeted covering, and of nonexcluded type. To each image of ramification points $x_{i} \in N$, we assign the number $\nu_{x_{i}}$, and to each $x_{i k}^{*} \in \pi^{-1}\left(x_{\imath}\right)$ we assign $\nu_{x_{i}} / r\left(x_{i k}^{*}\right)$ mentioned as above. Then there exist a simply connected domain $\tilde{M} \subset \widehat{C}$ and discontinuous groups of Möbius transformations $\Gamma, G$ with the following property.

$$
\Gamma \subset G, \quad \tilde{M} / \Gamma \simeq M, \quad \tilde{M} / G \simeq N .
$$

We denote by $p: \tilde{M} \rightarrow \tilde{M} / \Gamma$, and $p_{G}: \tilde{M} \rightarrow \tilde{M} / G$ the natural projections, then they are unramified except on $p^{-1}\left(\left\{x_{i k}^{*}\right\}\right)$ where

$$
b_{p}\left(\hat{x}_{i k}\right)=\nu_{x_{i k}^{*}} / r\left(x_{i k}^{*}\right)-1, \quad b_{p_{G}}\left(\hat{x}_{i k}\right)=\nu_{x_{i}}-1 \quad\left(\hat{x}_{i k} \in p^{-1}\left(x_{i k}^{*}\right)\right)
$$

and $p_{G}=\pi \circ p$.

Proof. By Klein-Poincaré limit circle theorem, we get a simply connected domain $\tilde{M} \subset \widehat{\boldsymbol{C}}$ and a discontinuous group $G$ with the property $\tilde{M} / G \simeq N$ and $b_{p G}\left(\hat{x}_{i k}\right)=\nu_{x_{i k}^{*}}-1$, except the following three cases a)-c).

a) $N=\hat{\boldsymbol{C}}$ and the image set of ramification points consists of one point on $N$.

b) $N=\widehat{\boldsymbol{C}}$ and the image set of ramification points consists of two points 
$\left\{x_{1}, x_{2}\right\} \subset N, \nu_{x_{1}} \neq \nu_{x_{2}}$.

c) $N=C$ and the image set of ramification points consists of one point on $N$.

But a) can not occur under the assumption. Indeed, $(M, \pi, N)$ is unlimited and finite-sheeted, so if $N=\widehat{\boldsymbol{C}}, M$ must be compact. Using Riemann-Hurwitz relation, $2(g-1)=2 n(0-1)+B$ ( $g$; genus of $M, n$; degree of the covering, $B=$ $\left.\sum_{x \in M} b_{\pi}(x)\right) . \quad B<n$ because the image set of ramification points consists of one point on $N$. The left-hand-side of the equality $\geqq-2$, and $-2 n+B<-n$, thus $n<2$ contradiction. In case b), we can take a common multiple of $\nu_{x_{1}}$ and $\nu_{x_{2}}$ instead of $\nu_{x_{1}}$ and $\nu_{x_{2}}$. Consequently, only the case c) remains excluded.

Now we will show that there exists an unlimited covering map $p$ onto $M$ which makes the diagram 1 commutative.

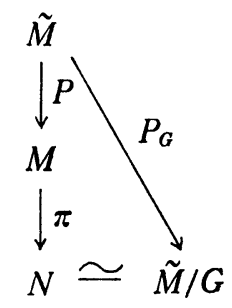

\section{diagram 1}

It is trivial that, if $x \in N$ is not an image of ramification points and we take $U_{x}$ as a neighbourhood of $x$ sufflciently small and take one component of $\pi^{-1}\left(U_{x}\right)$, then the restriction of $\pi^{-1} \circ p_{G}$ to a component of $p_{G}^{-1}\left(U_{x}\right)$ is comformal. If $x_{i} \in N$ is an image of ramification points, we may choose local coordinates $z, \hat{\zeta}, u$ vannishing at $x_{\imath}, \hat{x} \in p_{G}^{-1}\left(x_{\imath}\right), x^{*} \in \pi^{-1}\left(x_{\imath}\right)$ respectively, such that

$$
z=\hat{\zeta}^{k}, \quad f(z)=u^{l}
$$

$\left(k=\nu_{x_{i}}, l=b_{\pi}\left(x^{*}\right)+1\right.$, here $k$ is a multiple of $l$, and $f$ is conformal on a neighbourhood of $x_{2}$.).

We may write

$$
f(z)=\sum_{j=1}^{\infty} a_{\jmath} z^{\jmath} \quad\left(a_{1} \neq 0\right)
$$

and

$$
u^{l}=f(z)=f\left(\hat{\zeta}^{k}\right)=\hat{\zeta}^{k} \sum_{j=1}^{\infty} a^{j \hat{\zeta}^{k(\jmath-1)}}
$$

Since $a_{1} \neq 0$, we may take a single valued holomorphic function $g$ such that $g(\hat{\zeta})^{l}=\sum_{j=1}^{\infty} a_{j} \hat{\zeta}^{k(\jmath-1)}$ and $u=\hat{\zeta}^{k / l} g(\hat{\zeta})(k / l$ : integer). For the argument above, we see that we may define the map $p$ locally. Gluing the local maps, we get a holomorphic map $p: \tilde{M} \rightarrow M$.

Next we will show that $p$ is surjective. Let $\hat{x}_{0} \in \tilde{M}, x_{0}^{*} \in M$ be the begin- 
ning points of the gluing process of making $p$. For an arbitrary $x^{*} \in M$, we consider a curve $c:[0,1] \rightarrow M$ such that $c(0)=x_{0}^{*}, c(1)=x^{*}$, and $c([0,1])$ does not contain any image of ramification points on $M$ except $x^{*}$. The lift of $\pi(c)$ beginning at $\hat{x}_{0}$ ends at a point $\in \pi^{-1}\left(x^{*}\right)$.

The map $p$ is unlimited. Indeed, if a curve $c$ on $M$ does not contain any image of ramification points on $\tilde{M}$ except the ending point, it is easy to see that there is a lift beginning at any of $\in p^{-1}(c(0))$. Next we concider the case that $c$ is beginning at $p(\hat{x})$ where $\hat{x} \in \tilde{M}$ is one of the ramification points. Then, we may take $\hat{\zeta}, u$ as local coordinates of $\hat{x}, p(\hat{x})$ respectively, such that $u=$ $\hat{\zeta}^{n}(n \in N)$. Thus we get a lift beginning at $\hat{x}$. Consequently, for an arbitrary $c \subset M$, there is a lift beginning at any point $\in p^{-1}(c(0))$. Now we have shown that $p: M \rightarrow M$ is surjective, unlimited and making the diagram 1 commutative.

Take an arbitrary $\hat{x} \in \tilde{M}$ which is an unramified point of $p_{G}$ and fix it. For any $\hat{x}^{\prime} \in p^{-1} \circ p(\hat{x})$, there exists a $g \in G$ with $g(\hat{x})=\hat{x}^{\prime}$. Such an element $g$ has the following property (B).

$$
p \circ g\left(\hat{x}^{\prime \prime}\right)=p\left(\hat{x}^{\prime \prime}\right) \quad \text { for any } \quad \hat{x}^{\prime \prime} \in \tilde{M} .
$$

Indeed, if we take a curve $\hat{c}$ such that $\hat{c}(0)=\hat{x}, \hat{c}(1)=\hat{x}^{\prime \prime}$ and $\hat{c}$ has no ramification point of $p_{G}$ except $\hat{x}^{\prime \prime}$, then we have $p_{G}(\hat{c})=p_{G} \circ g(\hat{c})$. Having no ramification point of $\pi$ on $p(\hat{c})$ and $p \circ g(\hat{c})$ except $p(\hat{c}(1))$ and $p \circ g(\hat{c}(1))$ implies that $p \circ g\left(\hat{x}^{\prime \prime}\right)=p\left(\hat{x}^{\prime \prime}\right)$.

$$
\Gamma=\{g \in G \mid p(\hat{x})=p \circ g(\hat{x})\}
$$

form a subgroup of $G$. Indeed, if $g_{1}, g_{2} \in \Gamma$, property (B) implies that $p \circ g_{2}$ 。 $g_{1}(\hat{x})=p \circ g_{1}(\hat{x})=p(\hat{x})$. And $p\left(g_{1}^{-1}(\hat{x})\right)=p \circ g_{1}\left(g_{1}^{-1}(\hat{x})\right)=p(\hat{x})$.

We will show that $\tilde{M} / \Gamma \simeq M$. Let $\hat{x}_{1} \in \tilde{M}$ be an arbitrary unramified point of $p_{G}$. We consider a group

$$
\Gamma^{\prime}=\left\{g \in G \mid p\left(\hat{x}_{1}\right)=p \circ g\left(\hat{x}_{1}\right)\right\} .
$$

It is easy to see $\Gamma=\Gamma^{\prime}$ because of the property (B). Therefore the correspondence $\tilde{M} / \Gamma \rightarrow M\left([\gamma(\hat{x})]_{\gamma \in \Gamma^{\mapsto}} \mapsto p(\hat{x})\right)$ is well-defined and one-to-one on ah arbitrary unramified point of $p_{G}$. Even if $\hat{x} \in \tilde{M}$ is a ramification point of $p_{G}$, the correspondence is well-defined because of (B). If $[\gamma(\hat{x})]_{\gamma \in \Gamma} \neq[\gamma(\hat{y})]_{\gamma \in \Gamma}$, then $p(\hat{x}) \neq p(\hat{y})$. Indeed, assuming $[\gamma(\hat{x})]_{\gamma \in \Gamma} \neq[\gamma(\hat{y})]_{\gamma \in \Gamma}$ implies that there are neighbourhoods $U_{\hat{x}}, U_{\hat{y}}$ of $\hat{x}, \hat{y}$ respectively such that $p^{\prime}\left(U_{\hat{x}}\right) \cap p^{\prime}\left(U_{\hat{y}}\right)=\phi$, here $p^{\prime}$ : $\tilde{M} \rightarrow \tilde{M} / \Gamma$ is the natural projection. If $p(\hat{x})=p(\hat{y})$, there would be a point $z$ which is an image of unramified points of $p_{G}$ and $\in p\left(U_{\hat{x}}\right) \cap p\left(U_{\hat{y}}\right)$. For $\left\{\hat{z}_{x}, \hat{z}_{y}\right\}$ $\subset p^{-1}(z)$ with $\hat{z}_{x} \in U_{\hat{x}}, \hat{z}_{y} \in U_{\hat{y}}$, there would be a $\gamma \in \Gamma$ such that $\hat{z}_{x}=\gamma\left(\hat{z}_{y}\right)$. It contradicts $p^{\prime}\left(U_{\hat{x}}\right) \cap p^{\prime}\left(U_{\hat{y}}\right)=\phi$. Consequently, $p \circ p^{\prime-1}: \tilde{M} / \Gamma \rightarrow M$ is homeomorphic and holomorphic on the image set of unramified points of $p_{G}$. Thus the image points of ramification points are removable and $\tilde{M} / \Gamma \simeq M$.

DEFINITION 2. Let $G, \Gamma$ be discontinuous groups of Möbius transformations 
and $\Gamma \subset G$. We denote by $E_{G}(\Gamma)$ the group generated by $\Gamma$ and all of the elliptic elements of $G$. Trivially, $\Gamma \subset E_{G}(\Gamma) \subset G$.

\section{Theorem}

THEOREM. Let $(M, \pi, N)$ be ramified, unlimited, finite-sheeted, and of nonexcluded type. Let $\tilde{M} \subset \widehat{\boldsymbol{C}}, \Gamma, G$ be as in Lemma. Then for an arbitrary $T \in \operatorname{Aut}_{\pi}(M)$, there exists $T_{p} \in \operatorname{Aut}\left(\tilde{M} / E_{G}(\Gamma)\right)$ making the following diagram commutative. ( $\sigma$ is the natural projection.)

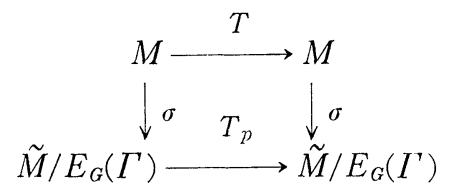

Here $\tilde{M}, G, \Gamma$ depend on $\left\{\nu_{x_{i}}\right\}$ but $\tilde{M} / E_{G}(\Gamma)$ does not, i.e, for another $\left\{\nu_{x_{i}}^{\prime}\right\}$, if we get $\tilde{M}^{\prime}, G^{\prime}, \Gamma^{\prime}$ instead of $\tilde{M}, G, \Gamma$ respectıvely, $\tilde{M}^{\prime} / E_{G\left(\Gamma^{\prime}\right)}^{\prime} \simeq \tilde{M} / E_{G\left(I^{\prime}\right)}$.

This assertion is a little stronger than that of the theorem in introduction, since $T \in \operatorname{Aut}_{\pi}(M)$ here. The following definition will be used in the proof of Theorem.

Definition 3. Let $x_{1}, \quad x_{2} \in \tilde{M} / \Gamma$ with $\sigma\left(x_{1}\right)=\sigma\left(x_{2}\right)$. We write $x_{1} \sim x_{2}$ if there exist curves $c_{1}, c_{2} \subset \tilde{M} / \Gamma$ satisfying the following conditions (1)-(4).

(1) $c_{1}, c_{2}$ share a ramification point of $\sigma$ at the beginning point.

(2) $x_{\imath}$ is the ending point of $c_{i}(i=1,2)$.

(3) $c_{\imath}$ has no ramification point except the beginnıng or ending point.

(4) $\sigma\left(c_{1}\right)=\sigma\left(c_{2}\right)$.

The symbol is not an equivalence relation because it is not transitive. Next we write $x_{1} \sim x_{2} \sim x_{3}$ if $x_{1} \sim x_{2}, x_{2} \sim x_{3}$. We write $x_{k} \cong x_{j}$ if $x_{k}$ and $x_{j}$ can be connected by finitely many $\sim$. Here $\simeq$ is an equivalence relation.

Proof. There exists a $\tilde{T} \in \operatorname{Aut}(\tilde{M})$ which makes the following diagram commutative for $T$.

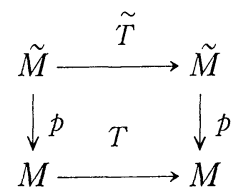

First we consider the case that $\tilde{M}=C$ or $\Delta$ (unit disk). We will show that, for an arbitrary elliptic element $\gamma \in G$, there exists an elliptic element $\gamma^{\prime} \in G$ such that $\gamma^{\prime} \circ \widetilde{T}=\widetilde{T} \circ \gamma$. We may assume $\gamma(0)=0$ without loss of generality. Denoting by $G_{0}$ all of the elements fixing 0 , we see that $G_{0}=\left\langle e^{2 \pi \imath / n} z\right\rangle$, here $n$ is the order of $G_{0}$. We take a disk $D_{0}$ centered at 0 satisfying the following property. 


$$
\begin{cases}g\left(D_{0}\right)=D_{0}, & g \in G_{0} \\ g\left(D_{0}\right) \cap D_{0}=\phi, & g \in G-G_{0}\end{cases}
$$

From the assumption about $T$, there exists $g_{0} \in G$ with $g_{0} \circ \widetilde{T}(0)=0$. We take a disk $D_{0}^{\prime}$ centered at 0 and satisfying

$$
D_{0}^{\prime} \subset \widetilde{T}^{-1} \circ g_{0}^{-1}\left(D_{0}\right) \cap D_{0} .
$$

If $\tilde{M} \simeq \Delta$, we may write $g_{0} \circ \tilde{T}(z)=e^{i \theta} z(0 \leqq \theta<2 \pi)$. If $\tilde{M} \simeq \boldsymbol{C}, g_{0} \circ \widetilde{T}(z)=a z(a \in \boldsymbol{C})$. In both cases, we may write $g_{0} \circ \tilde{T}(z)=a z(a \in C)$. We take $\zeta$ as a local coordinate of $p_{G}(0)$ satisfying $\left.p_{G}\right|_{D_{0}}: z \mapsto \zeta=z^{n}$. Then,

$$
\begin{gathered}
p_{G} \circ \tilde{T}\left(z_{1}\right)=p_{G} \circ g_{0} \circ \tilde{T}\left(z_{1}\right)=p_{G}\left(a z_{1}\right)=a^{n} z_{1}^{n}=a^{n}\left(\gamma\left(z_{1}\right)\right)^{n}=p_{G}\left(a \gamma\left(z_{1}\right)\right) \\
=p_{G} \circ g_{0} \circ \tilde{T}\left(\gamma\left(z_{1}\right)\right)=p_{G} \circ \tilde{T}\left(\gamma\left(z_{1}\right)\right) \quad \text { for } \quad z_{1} \in D_{0}^{\prime} .
\end{gathered}
$$

(Recall that $p_{G}: \tilde{M} \rightarrow \tilde{M} / G$ is the natural projection.)

Since $\widetilde{T}\left(z_{1}\right), \widetilde{T}\left(\gamma\left(z_{1}\right)\right) \in g_{0}^{-1}\left(D_{0}\right)$, there exists a $\gamma^{\prime} \in g_{0}^{-1} \circ G_{0} \circ g_{0}$ such that

$$
\gamma^{\prime} \circ \widetilde{T}\left(z_{1}\right)=\tilde{T}\left(\gamma\left(z_{1}\right)\right) \text {. }
$$

The order of $g_{0}^{-1} \circ G_{0} \circ g_{0}$ is $n$, so we take $n+2$ points $\left\{z_{i}\right\}$ on $D_{0}^{\prime}$ distinct each other, and considering as above we get $\gamma^{\prime} \circ \tilde{T}\left(z_{\imath}\right)=\tilde{T} \circ \gamma\left(z_{\imath}\right)$ for at least three points. Therefore, $\gamma^{\prime} \circ \tilde{T}=\tilde{T} \circ \gamma$.

For $\gamma \in \Gamma$, there exists a $\gamma^{\prime} \in \Gamma$ with $\gamma^{\prime} \circ \tilde{T}=\tilde{T} \circ \gamma$ because $\tilde{T}$ is a lift of $T \in$ $\operatorname{Aut}(\tilde{M} / \Gamma)$. Now we get $\tilde{T} E_{G}(\Gamma) \subset E_{G}(\Gamma) \tilde{T}$ and $T_{p}$ is well-defined. Indeed,

$$
\begin{aligned}
& \sigma \circ p\left(z_{1}\right)=\sigma \circ p\left(z_{2}\right), \quad z_{1}, z_{2} \in \tilde{M} \Rightarrow \text { there exists } g \in E_{G}(\Gamma) \text { with } g\left(z_{1}\right)=z_{2} \Rightarrow \\
& \tilde{T}\left(z_{2}\right)=\tilde{T} \circ g\left(z_{1}\right)=g^{\prime} \circ \tilde{T}\left(z_{1}\right), \quad g^{\prime} \in E_{G}(\Gamma) \Rightarrow \sigma \circ p \circ \tilde{T}\left(z_{2}\right)=\sigma \circ p \circ \tilde{T}\left(z_{1}\right) .
\end{aligned}
$$

It is easy to see $T_{p}$ is bijective. Since $\sigma \circ p$ is continuous and an open mapping, $T_{p}$ is a homeomorphism. When a point $q \in \tilde{M} / E_{G}(\Gamma)$ is not an image of ramification points of $\sigma \circ p, T_{p}$ is holomorphic on a neighbourhood of $q$. When $q$ is an image of ramification points, $q$ is a removable singular point because $T_{p}$ is a homeomorphism. Now we see that $T_{p} \in \operatorname{Aut}\left(\tilde{M} / E_{G}(\Gamma)\right)$.

Next we consider the case $\tilde{M}=\widehat{\boldsymbol{C}}$. In this case, every element of $G$ is elliptic, so $E_{G}(\Gamma)=G$. All of the signatures and cardinalities of discontinious group $G$ that could possibly act on $\widehat{\boldsymbol{C}}$ is classified. (see [1], [2])

$\begin{array}{cc}\text { signature of } G & |G| \\ (0 ; \cdots) & 1 \\ (0 ; \nu, \nu) & \nu(2 \leqq \nu<\infty) \\ (0 ; 2,2, \nu) & 2 \nu(2 \leqq \nu<\infty) \\ (0 ; 2,3,3) & 12 \\ (0 ; 2,3,4) & 24 \\ (0 ; 2,3,5) & 60\end{array}$


If the signature of $G$ is $(0 ; \nu, \nu), G$ is cyclic. Without loss of generality, we may assume that the fixed points of $g \in G$ are $0, \infty$. Thus we may write $g(z)=e^{2 \pi \imath / \nu} z$. Since $\tilde{T}$ the lift of $T \in \operatorname{Aut}(M)$ also fixes $0, \infty, \widetilde{T}(z)=a z(a \in C)$. We get $\tilde{T} \circ g=g \circ \tilde{T}$. If the signature of $G$ is $(0 ; 2,2, \nu)$, we consider a meromorphic function on $\tilde{M} h=p_{G}-p_{G} \circ \tilde{T}$. Assuming $h \notin C$, we will get contradiction. Without loss of generality, we may assume that $\infty \in N=\widehat{\boldsymbol{C}}$ is not an image of ramification points of $p_{G}$. Then $\#^{-1}(\infty) \leqq 4 \nu$, and so deg $h \leqq 4 \nu$ (here $\operatorname{deg} h$ is the degree of $(\tilde{M}, h, \hat{\boldsymbol{C}})$ ). Next we consider $h^{-1}(0)$. We denote by $x_{1}, x_{2}, x_{3}$ the points on $N$ corresponding to $2,2, \nu$ of the signature $(0 ; 2,2, \nu)$ respectively. Let $z_{1} \in p_{G}^{-1}\left(x_{1}\right)$. It is one of zeroes of $h$. Taking suitable local coordinate $\zeta, z$, $u$ of $z_{1}, p_{G}\left(z_{1}\right), \tilde{T}\left(z_{1}\right)$ respectively, we may write $z=\zeta^{2}, z=u^{2}$, $u(\zeta)=\tilde{T}(\zeta)=a_{1} \zeta+a_{2} \zeta^{2}+\cdots\left(a_{1} \neq 0\right)$. Then $h(\zeta)=\zeta^{2}-\left(a_{1} \zeta+\cdots\right)^{2}$. Thus the order of $z_{1}$ as a zero of $h$ is $\geqq 2$. Since $\#_{\phi_{G}^{-1}}\left(x_{1}\right)=\nu$, the total sum of the order of all of the points $\in p_{G}^{-1}\left(x_{1}\right)$ as zeroes of $h$ is $\geqq 2 \nu$. The same consideration about $x_{2}, x_{3}$ as above leads us to the conclusion that $\operatorname{deg} h \geqq 6 \nu$, contradiction. Consequently, $h \equiv 0$. Thus we get $p_{G}=p_{G} \circ \tilde{T}$ and $\pi=\pi \circ T$. If it is the case remaining, we consider same as above and get $\pi=\pi \circ T$.

Finally, we will show the uniqueness of $\tilde{M} / E_{G}(\Gamma)$. We assigned $\nu_{x_{2}}$ a common multiple of all of the ramification numbers of $\pi^{-1}\left(x_{\imath}\right)$ to each image of ramification points $x_{i} \in N$. We may take another common multiple $\nu_{x_{i}}^{\prime}$ instead of each $\nu_{x_{i}}$ and get $\tilde{M}^{\prime}, G^{\prime}, \Gamma^{\prime}$ instsad of $\tilde{M}, G, \Gamma$. We will show that $\tilde{M} / E_{G}(\Gamma) \simeq \tilde{M}^{\prime} / E_{G^{\prime}}\left(\Gamma^{\prime}\right)$. First, $\tilde{M} / \Gamma \simeq M$ and $\tilde{M}^{\prime} / \Gamma^{\prime} \simeq M$, thus $\tilde{M} / \Gamma \simeq \tilde{M}^{\prime} / \Gamma^{\prime}$. We denote by $f: \tilde{M} / \Gamma \rightarrow \tilde{M}^{\prime} / \Gamma^{\prime}$ the conformal map. We will show that $\sigma^{\prime} \circ f \circ \sigma^{-1}$ is well-defined, here $\sigma^{\prime}: \tilde{M}^{\prime} / \Gamma^{\prime} \rightarrow \tilde{M}^{\prime} / E_{G^{\prime}}\left(\Gamma^{\prime}\right)$ is the natural projection.

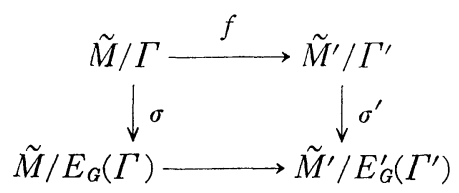

We will show $\sigma\left(x_{1}\right)=\sigma\left(x_{2}\right)\left(x_{1}, x_{2} \in \tilde{M} / \Gamma\right) \Rightarrow x_{1} \simeq x_{2}$, where $\simeq$ is the equivalence relation of definition 3. If $\sigma\left(x_{1}\right)=\sigma\left(x_{2}\right)$, for $\hat{z}_{1} \in p^{-1}\left(x_{1}\right), \hat{z}_{2} \in p^{-1}\left(x_{2}\right)$, we may write

$$
\begin{aligned}
& \hat{z}_{2}=a_{k} \circ \cdots \circ a_{2} \circ a_{1}\left(\hat{z}_{1}\right) \\
& a_{j} \in \Gamma \quad \text { or } \quad a_{j} \in\{\text { elliptic elements of } G\}-\Gamma \quad(\jmath=1, \cdots, k) .
\end{aligned}
$$

If $a_{1} \in \Gamma, a_{1}\left(\hat{z}_{1}\right)$ and $\hat{z}_{1}$ are projected to the same point on $\tilde{M} / \Gamma$. If $a_{1} \in$ elliptic elements of $G\}-\Gamma$, we may draw a curve $c \subset \tilde{M}$ such that $c(0) \in\{$ fixed points of $\left.a_{1}\right\}, c(1)=\hat{z}_{1}$ and $c$ has no fixed points of elliptic elements except $c(0), c(1)$. Then $p(c)$ and $p\left(a_{1}(c)\right)$ have the same beginning point which is a ramification point of $\sigma$, ending at $p\left(\hat{z}_{1}\right), p\left(a_{1}\left(\hat{z}_{1}\right)\right)$ respectively and $\sigma(p(c))=\sigma\left(p\left(a_{1}(c)\right)\right)$. Therefore, $x_{1} \sim p\left(a_{1}\left(\hat{z}_{1}\right)\right)$. The same consideration about $a_{2}, \cdots, a_{k}$ as above leads us to the conclusion that $x_{1} \cong x_{2}$.

Next we will show $x_{1} \sim x_{2} \Rightarrow \sigma^{\prime} \circ f\left(x_{1}\right)=\sigma^{\prime} \circ f\left(x_{2}\right)$. By the definition of $\sim$, we 
have curves $c_{1}, c_{2}$ satisfying the conditions (1)-(4) of definition 3 . We may take the same coordinate neighbourhood of $\sigma^{\prime} \circ f(x)$ and $q^{\prime} \circ \sigma^{\prime} \circ f(x)\left(q^{\prime}: \tilde{M}^{\prime} / E_{G^{\prime}}\left(\Gamma^{\prime}\right) \rightarrow\right.$ $\tilde{M}^{\prime} / G^{\prime}$ is the natural projection) because $q^{\prime}$ is unramified. We denote it by $U$. We denote by $\pi^{\prime}$ the composition of the conformal map $\tilde{M}^{\prime} / \Gamma^{\prime} \rightarrow M$ and $\pi: M$ $\rightarrow N$. Then,

Thus,

$$
\pi^{\prime} \circ f\left(c_{1}\right)=\pi^{\prime} \circ f\left(c_{2}\right)
$$

$$
\sigma^{\prime} \circ f\left(c_{1}\right) \cap U=\sigma^{\prime} \circ f\left(c_{2}\right) \cap U
$$

We may take two holomorphic maps as $\left.\sigma^{\prime} \circ f \circ \sigma^{-1}\right|_{\sigma\left(c_{1}\right)}$ taking $c_{1}$ or $c_{2}$ as $\sigma^{-1}\left(\sigma\left(c_{1}\right)\right)$. But by the uniqueness theorem and $(*)$, we see that $\sigma^{\prime} \circ f\left(c_{1}\right)=\sigma^{\prime} \circ f\left(c_{2}\right)$. Therefore, we get $\sigma^{\prime} \circ f\left(x_{1}\right)=\sigma^{\prime} \circ f\left(x_{2}\right)$. Now we have shown that $x_{1} \sim x_{2} \Rightarrow \sigma^{\prime}$ 。 $f\left(x_{1}\right)=\sigma^{\prime} \circ f\left(x_{2}\right)$. Of course, it implies that $x_{1} \simeq x_{2} \Rightarrow \sigma^{\prime} \circ f\left(x_{1}\right)=\sigma^{\prime} \circ f\left(x_{2}\right)$. For $\sigma\left(x_{1}\right)=\sigma\left(x_{2}\right) \Rightarrow x_{1} \simeq x_{2}$ as we have shown, $\sigma\left(x_{1}\right)=\sigma\left(x_{2}\right) \Rightarrow \sigma^{\prime} \circ f\left(x_{1}\right)=\sigma^{\prime} \circ f\left(x_{2}\right)$. Thus $\sigma^{\prime} \circ f \circ \sigma^{-1}$ is well-defined. Considering $\sigma \circ f \circ \sigma^{\prime-1}$, it is easy to see that $\sigma^{\prime} \circ f \circ \sigma^{-1}$ is bijective. Therefore, $\sigma^{\prime} \circ f \circ \sigma^{-1}$ is homeomorphic and locally conformal except on the images of ramification points of $\sigma$. Thus, they are removable singular points and we get $\tilde{M} / E_{G}(\Gamma) \simeq \tilde{M}^{\prime} / E_{G^{\prime}}\left(\Gamma^{\prime}\right)$.

Now we know that any $T \in \operatorname{Aut}_{\pi}(M)$ can always be projected to $T_{p} \in$ $\operatorname{Aut}\left(\tilde{M} / E_{G}(\Gamma)\right)$. But it is not always the case that there is a $T_{*} \in \operatorname{Aut}(N)$ such that $\pi \circ T=T_{*} \circ \pi$. We see it by the following example.

Example. First we consider the Riemann sphere $\hat{\boldsymbol{C}}$. From $\hat{\boldsymbol{C}}$, we remove 8 disks each of which is centered at $e^{\pi \imath(2 k+1) / 8}(k=0,1, \cdots, 7)$ and has the same radius $\varepsilon<\sin (\pi / 8)$. Then we get a Riemann surface with boundaries. We denote it by $D$. Consider now two copies $D$ and $D^{\prime}$ of $D$ and construct a compact Riemann surface $M=D \cup D^{\prime}$ known as the double of $D$. The genus of $M$ is 7 . Let $z$ be the usual coordinate on $D$, and consider a conformal map $u: z \mapsto-z$, $z \in D$. Here $u$ can be extended to a conformal map on $M$. We also denote it by $u: M \rightarrow M$. An anti-conformal map $a: z \mapsto \bar{z}, z \in D$ can be extended to an anti-conformal map on $M$. We also denote it by $a: M \rightarrow M$. Let $\}$ denote the reflection (the anti-conformal involution) on $M$. Then $v=a \circ \mathrm{\jmath}$ is conformal, $u^{2}=\mathrm{id}, v^{2}=\mathrm{id}, u \circ v=v \circ u$ and $(u \circ v)^{2}=\mathrm{id}$. Thus the order of the group $\langle u, v\rangle$ is 4 , and the degree of the covering map $\pi: M \rightarrow M /\langle u, v\rangle$ is 4 . Here the ramification points are $0, \infty \in D \subset M$ and the corresponding points of these on $D^{\prime} \subset$ $M$. The ramification number of these are all 2. Using Riemann-Hurwitz formula, we see that the genus of $N=M /\langle u, v\rangle$ is 2 .

Let $T: z \mapsto e^{\pi \imath / 4} z, z \in D$. It can be extended to a conformal map on $M$. We also denote it by $T$. Since $T$ fixes all of the ramification points of $\pi$ on $M$, we see that $T \in \operatorname{Aut}_{\pi}(M)$. Let $2^{\prime} \in D^{\prime}$ denote the point corresponding to $2 \in D$. Then $\pi(2)=\pi\left(2^{\prime}\right)$ because $v(2)=2^{\prime}$. Here $T(2)=2 e^{\pi \imath / 4}, T\left(2^{\prime}\right)=2^{\prime} e^{\pi \imath / 4}$ and $\pi\left(2 e^{\pi \imath / 4}\right)$ $\neq \pi\left(2^{\prime} e^{\pi \imath / 4}\right)$. Therefore, $\pi \circ T \circ \pi^{-1}$ is not well-defined. Now we see that there is no $T_{*} \in \operatorname{Aut}(N)$ which makes the following diagram commutative. 


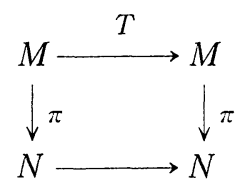

From our theorem, we get next two corollaries.

COROLLARY 1. If $(M, \pi, N)$ satisfies the same assumptions of the theorem and the degree of the map is prime, then for an arbitrary $T \in \mathrm{Aut}_{\pi}(M)$ there exists a $T_{*} \in \operatorname{Aut}(N)$ with $T_{*} \circ \pi=\pi \circ T$.

Proof. $[G: \Gamma]=\left[G: E_{G}(\Gamma)\right] \times\left[E_{G}(\Gamma): \Gamma\right],[G: \Gamma]$ is prime, thus $E_{G}(\Gamma)$ $=G$.

COROLLARY 2. If $(M, \pi, N)$ satisfies the same assumptions of the theorem and $N$ is simply connected, then for an arbitrary $T \in \operatorname{Aut}_{\pi}(M)$ there exists a $T_{*}$ $\in \operatorname{Aut}(N)$ with $T_{* \circ} \pi=\pi \circ T$.

Proof. $\tilde{M} / E_{G}(\Gamma) \cong N$ because $\tilde{M} / E_{G}(\Gamma) \rightarrow N$ is unlimited and unramified.

We exhibit one of properties of $\tilde{M} / E_{G}(\Gamma)$ here. If coverings $\left(M, \sigma^{\prime}, S^{\prime}\right)$, $\left(S^{\prime}, q^{\prime}, N\right)$ are unlimited, making the diagram A commutative and $\left(S^{\prime}, q^{\prime}, N\right)$ is unramified, then the unlimited unramified covering $\left(\tilde{M} / E_{G}(\Gamma), \sigma^{\prime \prime}, S^{\prime}\right)$ makes the diagram B commutative. Indeed, by the argument of the lemma, we get a discontinuous group $K$ such that $\tilde{M} / K \simeq S^{\prime}, \quad \Gamma \subset K \subset G$. Then an arbitrary elliptic element $\in G$ belongs to $K$ because $q^{\prime}: S^{\prime} \rightarrow N$ is unramified. Therefore, $E_{G}(\Gamma) \subset K$.
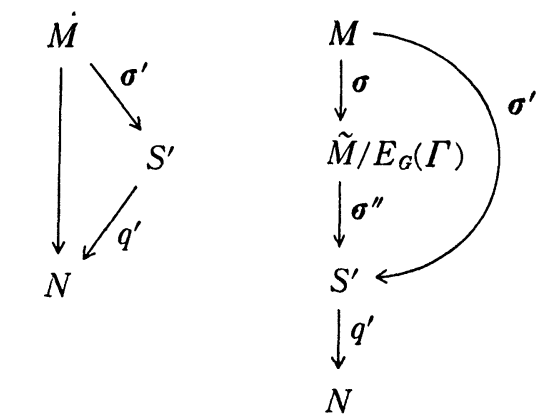

diagram $\mathrm{A}$

diagram $\mathrm{B}$

\section{REFERENCES}

[1] H. M. FARKAS AND I. KRA, Riemann Surfaces, Springer-Verlag, 1980.

[2] L. FORD, Automorphic Functions, McGraw-Hill, 1929. 
[3] I. KRA, Automorphic Forms and Kleinian Groups, W.A. Benjamın Inc, 1972.

[4] J. LEHNER, Discontinuous Groups and Automorphic Functions, Mathematical Surveys, 8, American Mathematical Society, 1964

[5] H. Martens, Observations on morphisms of closed Riemann surfaces, Bull. London Math. Soc., 10 (1978), 209-212.

Department of Mathematics

TOKYO INSTITUTE OF TECHNOLOGY

OH-OKayama, Meguro-Ku, TOKyo, 152

JAPAN 